\title{
A Radio Frequency Identification Technology- Based Positioning and Interference Suppression System for Library Books
}

\author{
https://doi.org/10.3991/ijoe.v12i11.6236 \\ Zhang Xiao-guang, Zhao Yi-jie, Zhao Wei \\ Hebei North University, Hebei, China
}

\begin{abstract}
Large scale path loss modeling plays a fundamental role in designing both fixed and mobile radio systems. Wireless system are complex systems, so that prediction of the radio coverage is not done in an exact manner. Therefore before setting the system we need to choose a proper model of predicting path loss and interference. These proper methods depend upon the system environment, desired radio coverage and system frequency. In this paper of predicting path loss and interference analysis of UHF-RFID, we try to eliminate the problems associated of positioning and interference suppression system of library books to RFID systems. Simulation results show that the proposed method can thus improve overall system performance substantially.
\end{abstract}

Index Terms-positioning, interference suppression system, library books, radio frequency identification technology

\section{INTRODUCTION}

In context of wireless engineering, one of the most discussed visions is the "Internet of things" IoT. The main theme of the IoT is that any place, person or object can be part of a virtual system, upon which any other has access. In this way, anything becomes a living actor virtually. RFID systems are the good candidates for "Internet of Things" which are diffusing everywhere for automatic identification applications. Each object for the purpose of identification and wireless communication hold a small RFID tag containing all the necessary information in accordance to a company or unit structure [1-3].

EZFID is an abbreviation of radio frequency identification. RFID tags are attached with the object to be identified with RFC reader [4]. The main function of readers is to scan the entire tagged substance without any line of sight environment. There are two main components of EZFID system i.e. a reader and a tag. The Antenna is plugged to the reader with a transmitter-receiver, while a tag consists of memory, antenna and an integrated circuit. Object information is embedding in the tag affix to the object to be identified. Tag may contain several type of information like object identification number, size, types and other information related to the object. Furthermore each tag has an identifier to identify objects. Electromagnetic waves are used for communication between reader and tags [5]. Each individual tagged item can get a unique identification number affixed by a tag containing many additional details about the object. RFID system is a replacement toward traditional bar code system for identifying objects. In bar-coded system objects requires a direct line of sight LOS and one item or object can be read at a time. This will overcome in RFID system, as RFID system network contains multiple tags and tagged object that can read simultaneously. This system also provides us a unique read property in which no line of sight is required.

According to different power support methods, RFID tags can be classified into two types: active RFID and passive RFID tags. Active tags contain batteries while passive tags need external power to awake the signals. The maximum working range of passive tags is 10 meters. RFC systems have two normal working patterns: RTF (Reader talks first) and TTF (tags talk first). In RTF reader sends commands to tags first, tags will reply when receive the command where as in TTF tags will directly send messages to reader in a range. When EZFID reader sends command to tags, tags respond simultaneously, the signals will interfere with each other. This is typically called a collision and the result of the communication is failed. If one reader communicates with more than one tag, anticollision methods must be applied. Due to the characters of RFID tags, the anti-collision has limited resources such as limited computation power and status information etc. collision might be difficult to detect because of the vary signal strengths between tags.

Radio Frequency Identification (RFID) systems are gaining popularity since some big companies in the USA, such as Wal-Mart, had mandated the use of RFID tags in their respective supply chains. With increasing interest in deploying RFID system on large scale, problems emerge, and one of them is the reader collision problem. Reader collision problems mainly occur in a dense reader environment, where several readers try to interrogate tags at the same time in same vicinity. The read results can be unsatisfactory with read times and an unacceptable level of misreads. In Europe, the fear of RFID disrupting nonRFID devices operating in the same frequency band as RFID systems has prompted the introduction of the concept of a "Listen before Talk" (LBT) provision for RFID systems, causing some uncertainties over the feasibility of RFID global deployment [6-8].

During last decade UHF technology proved to be the most promising solution especially for large scale RFID applications. This will enabled to tag a large number of objects with smart labels. This chapter explains the title of this thesis, which is "Path loss Propagation and Interference Mitigation in UHF-RFID systems". The back ground of this research topic is Radio Frequency Identification is presented. The motivation, Problem statements, Goals and technical specifications are discussed, followed by the elaboration of the thesis structure. 


\section{OVERVIEW}

In a great variety of contexts the automatic identification is used to improve process performance, i.e. from the access control to the traceability of items. There are several reasons such as the reduction of both times required completing a task, and number of mistakes, the precision and repeatability and so on: it's worth noting that all of them allow reducing costs and improving service. RFID in context of wireless communication technology also represents it for automatic identification purpose of the items. That's make this technology more promising in the world.

RFID is an emerging technology that has potential to make great economic impact on many industries. This technology also used in AIDC for object identifications. Without the need of line-of-sight communication, RFID systems identify physical objects automatically because of radio waves. Michael faraday in 1840 s opens the new horizons for radio waves and its radio properties that lead for industries to mainly focus on the entire technology in recent era. Radio technology and development of radar can emerge the development of RFID, so both radar and radio waves technology is a part of a typical RFID system. Then it latter used in Second World War first time for military applications. For identification purpose radio frequency is installed on Allied aircrafts. For the sake of this paradigm this technology become popular by the name IFF (identifies friend or Foe) [9].

Before discussing some more aspect in RFID system it is important to know about the background of RFID. The history of RFID shows a clear sequence of its development and related technologies.

EZFID systems are based on the man's understanding of the electromagnetic world. The main development of RFID starts in the 1950s after expansion of radar and radio technology. In the late 1960 s radio frequency become popular in nuclear and other hazardous materials. This will open the new ways for engineer to more work on it. Following itemizations are the best description of the significance development of RFID.

(1) The first continuous radio wave is attained by Ernst F.W Alexanderson in 1906. The accomplishment critically accelerated the process of the birth of radar, which is the original application of RFID.

(2) In 1935, Sir Robert Alexander Waston-watt invents first RFID system. This system helps to communicate between friendly and unfriendly aircraft by the signal transmission.

(3) Harry stock-man gives a new research in 1948. This research was related to theoretical foundation of RFID technology. This was a landmark for RFID new researchers. A tag is used to read and write data to its associated memory. When a tag entered to its interrogation zone the data can be transferred from tag memory to the reader.

(1) Classification of RFID tags:

Tags are also classified with respect to their capabilities. It may be read-only, write only or re-write only for further data recording). Narayanan et al, in 2005 derived different classes of tags as shown in figure 1. As a name we know that the physical feature of RFID tags is size, shapes, and weight. Some other are:

(1) Smart tags associated with layers type equipment; It may be a piece of paper
(2) Tiny tags are may embed to flat objects like keys and clothes.

(3)Plastic disk type tags can mostly be used for durable object and may also use for tough environment for example pallets tagging use in open air.

(4) Tags are also being classified in term of their capabilities and performance. Following are the list for tags capabilities.

(5)Anti-collision capability of tag; This enable for reader to read all tags within the zone.

(6) Power source and Tag durability.

(7) Tags data writing capabilities such as write once or many times onto tags memory.

(8)Coupling mechanism such as inductive, magnetic, capacitive and backscatter. The coupling mechanism is used to determine power sharing method and tag information

(9) Tag may communicate in full duplex or half duplex way.

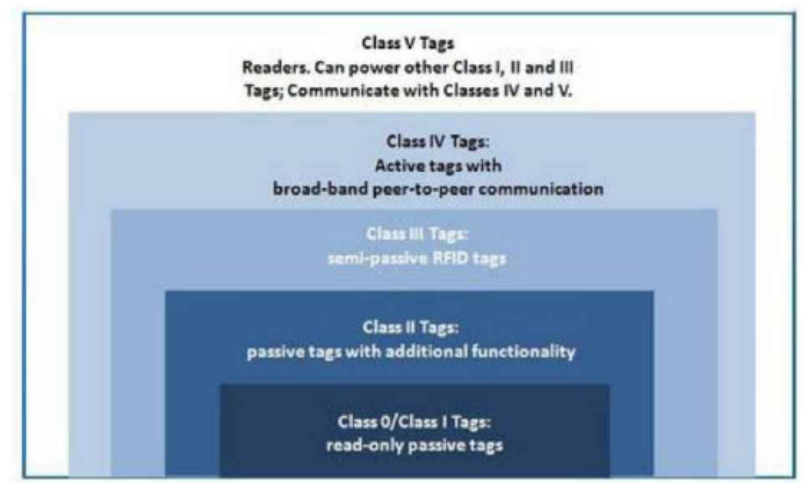

Figure 1. Classes of RFID tags

RFID have different elements of tags and readers. Here we present different tags factors as shown in figure 2. Generally consist of a simple circuit like resistor-inductor circuit. In simple term RF front end is a generic term used for all types of circuit between antenna and the first intermediate frequency IF stage. In same manner RF front-end is an interface between antenna and signal processing unit in RFC. RF front end circuit main responsibilities are resets and connection, connection with external antenna, voltage regulators and implementing modulator.

Control logic control the main function i.e. the error checking and parity/ CRC checkers, controllers, anticollision algorithms, command decoders and data encoder. Advance RFID ICs also embed the mechanism of data encryption and even tamper-proofing hardware.

EEPROM (Electrically erasable and programmable read-only memory) provide functionality to tags to be $\mathrm{read} /$ write. The tags that cannot be programmed use state mechanism or read-only memories ROM) to store or generate information. The tag antenna receives and reflects $\mathrm{RF}$ waves coming from the reader antenna. The antenna is the most significant part of the tag, on which the tag size is determined. Antenna is designed for a particular frequency and customized for the application. Communication interface provides read a mean of interaction with an eternal host system by transferring data, passing or responding to instructions. Different types of reader have different communication interface and functionality. 


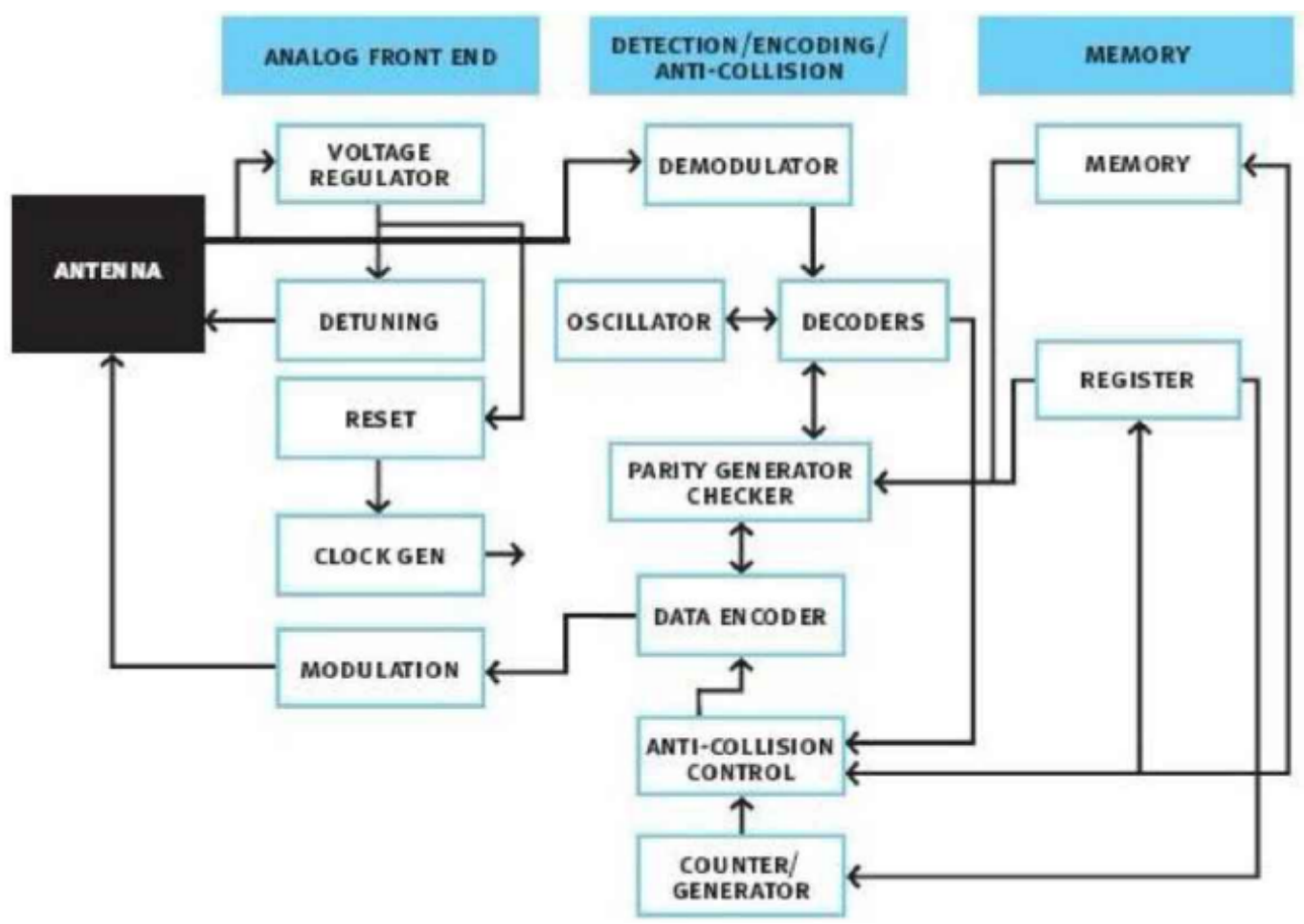

Figure 2. Elements of RFID tags with attached antenna

Serial reader uses a RS232 serial port to communicate with host computer and transfer data or command executed by the user or application. The limitations of this type of reader are lower data transfer rate and have a lack of cable in length. But the reliability is a main feature of this type of reader. Network reader operates wirelessly or connected with a computer through wired, so it will treat as a network device in a dense environment. For this kind of reader length is not a matter but reliability is not as better as of serial reader. Stationary reader mounted on a wall or on a static place in reading zone. This type of reader may be embedded with moving objects such as vehicle. Wall mart uses an RFID printer; is a type of stationary reader that is use to write on tags and print a barcode. Handheld reader has an incorporated antenna on it. Handheld device operate freely as a handheld unit.

\section{MeTHOD AND ALGORITHM}

In RFID field, there is a simplest way for positioning, which only depends on the tags detecting by readers without a distance measurement. In this case, there must be a large amount of tags so that the reader can roughly know the position because it knows which tag (or which tags) it can read. However, people prefer some more accurate positioning methods in practice. The current scheme of RFID accurate positioning can be classified into four categories which are TOA (time of arrival), RSSI (Received Signal Strength Indication), TDoA (time Difference of Arrival and AoA (Angle of Arrival).

In telecommunications, RSSI is a method of distance measurement is accomplished by detecting the degradation of signal strength, since that the level of the signal degradation is almost inversely proportional to the distance.

RSSI scheme is relatively easy and simple to implement. However, the accuracy cannot be guaranteed due to its vulnerability to reflection and multipath interference.
ToA used to measure the distance between transmitter and receiver by obtaining the time consumed during the signal traveling. In order to calculate the distance, the speed of light in a certain medium (usually can be defined as vacuum in the air condition) or the frequency of the carrier wave is required. That is calculated through. Note that ToA uses the absolute time, which means the exact time when the signal departure and arrival should be known. Because the accuracy of time is very important in ToA method, a synchronous time on both a receiver and transmitter becomes extremely necessary which also adds the complication to the implementation.

AoA is a distance measurement by detecting the direction of the propagation of the signals when it incident on an antenna array. With the help of TDoA at individual elements of the antenna array, the AoA can be calculating from these time delays. For instance, there is a receiving antenna array contain two elements spaced apart by a half of the wavelength. If the signal the signal issued from the transmitter incident upon the array by going along with bore sight (the optical axis of a directional antenna), it will arrive at each antenna simultaneously which stand for a 00 between two antenna phase-differences measured elements, equivalent to a $00 \mathrm{AoA}$. In this way, a 1800 phase difference will be generated between the two antenna elements due to a broadside incident signal and the half wavelength distance between which correspond to a 900 AoA in figure 3.

In the context of UHF' RFID systems, there are mainly three categories of tags: passive tag, BAP tags, and active tags. In both passive and BAP tags, the communication with the reader is managed modulating the incident electromagnetic wave; passive tags differ because they also feed their internal circuits using part of the incident energy. As said, the greatest interest is concentrated on passive tags, because, despite of a simpler architecture, their cost allows the massive employment. Nevertheless, the effi- 


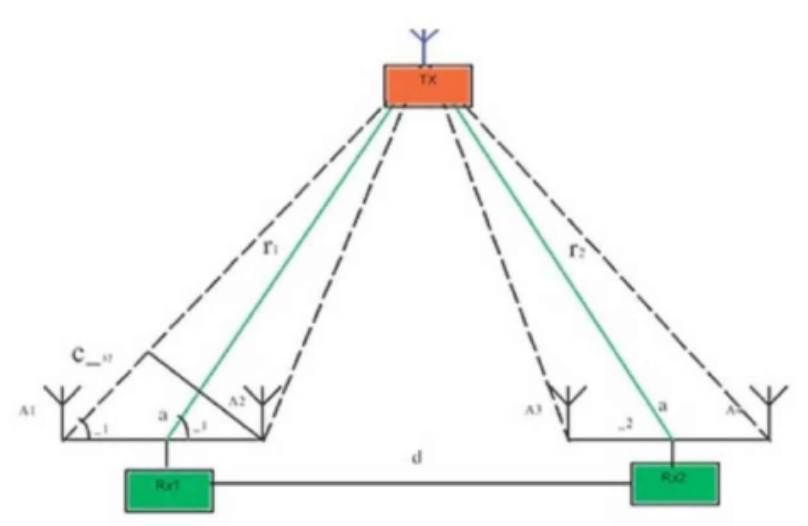

Figure 3. Two elements spaced apart by a half of the wavelength-AoA

ciency of the mechanism of transmission and retransmission is vital to ensure the proper functionality of the whole system.

In particular, the power that reaches tags' antenna is a fundamental parameter, because it influences both the activation of the circuits, and the power level of the backscattered signal. Data stored on tags must be sent to readers in a reliable manner. Encoding this data and transmitting it over a modulated signal are two critical components of reliable communications. The choice of coding and modulation schemes determines the bandwidth, integrity and power consumption of tag-to-reader communications.

The power and modulation capabilities of tags restrict which coding and modulation schemes and appropriate for RFC systems. Bandwidth is another limiting factor. Although readers may transmit at high power, government restrictions typically limit the side band resulting from modulation. However, since passive tags do not actively transmit a signal, the encoding on the backward channel is not subject to these restrictions and may occupy a high bandwidth. Our thesis focus is not towards coupling so we close this discussion here with explaining the three criteria for selecting RFC coding technique.

(1) The code should maintain power to the tag as much as possible.

(2) The code should not consume too much bandwidth.

(3) Collisions must be detected.

The equation of basic function is as equation (1) as follows:

$$
\partial_{j}\left(C_{i j k l} \partial_{k} u_{l}+e_{k i j} \partial_{k} \varphi\right)-\rho \ddot{u}_{i}=0
$$

Under the linear relationship, basic equation is shown in equation (2):

$$
\partial_{j}\left(e_{i j k l} \partial_{k} u_{l}-\eta_{k i j} \partial_{k} \varphi\right)=0 \text { (2) }
$$

The linear differential equation can be expressed into the following simplified forms:

$$
\begin{aligned}
L(\nabla, \omega) f(x, \omega) & =0, \\
L(\nabla, \omega) & =T(\nabla)+\omega^{2} \rho \mathrm{J}
\end{aligned}
$$

In which,

$$
T(\nabla)=\left\|\begin{array}{cc}
T_{i k}(\nabla) & t_{i}(\nabla) \\
t_{k}^{T}(\nabla) & -\tau(\nabla)
\end{array}\right\|, \mathrm{J}=\left\|\begin{array}{cc}
\delta_{i k} & 0 \\
0 & 0
\end{array}\right\|,
$$

$$
\begin{gathered}
f(x, \omega)=\left\|\begin{array}{c}
u_{k}(x, \omega) \\
\varphi(x, \omega)
\end{array}\right\| \\
T_{i k}(\nabla)=\partial_{j} C_{i j k l} \partial_{l}, t_{i}(\nabla)=\partial_{j} e_{i j k} \partial_{k}, \\
\tau(\nabla)=\partial_{i} \eta_{i k} \partial_{k}
\end{gathered}
$$

Consider an infinite situation, we have the equation (5) in the following:

$$
L^{0}=\left\|\begin{array}{cc}
C_{i j k l}^{0} & e_{k i j}^{0} \\
e_{i k l}^{0 T} & -\eta_{i k}^{0}
\end{array}\right\|
$$

Consider the propagation, instead the equation (5) with the following form:

$$
\begin{gathered}
C(\mathrm{x})=C^{0}+C^{1}(\mathrm{x}), \quad e(\mathrm{x})=e^{0}+e^{1}(\mathrm{x}) \\
\eta(\mathrm{x})=\eta^{0}+\eta^{1}(\mathrm{x}), \quad \rho(\mathrm{x})=\rho_{0}+\rho_{1}(\mathrm{x})
\end{gathered}
$$

Then we have equation (7) to (9):

$$
\begin{aligned}
& C^{1}=C-C^{0}, \quad e^{1}=e-e^{0} \\
& \quad \eta^{1}=\eta-\eta^{0}, \quad \rho_{1}=\rho-\rho_{0}
\end{aligned}
$$

The containing inclusions can be simplified into the following integral equation set:

$$
\begin{aligned}
& f(x, \omega)=f^{0}(x, \omega)+\int_{V} S\left(x-x^{\prime}\right)\left(\mathrm{L}^{1} F\left(\mathrm{y}^{\prime}\right)\right. \\
& \left.+\rho_{1} \omega^{2} \mathbf{g}(R) \mathrm{T}_{1} f\left(\mathrm{y}^{\prime}\right)\right] S\left(\mathrm{y}^{\prime}\right) \mathrm{dy}^{\prime}
\end{aligned}
$$

In view of the following relationship

$$
\frac{1}{2 \pi} \int_{-\infty}^{\infty} e^{-i k_{3} x_{3}^{\prime}} d x_{3}^{\prime}=\delta\left(k_{3}\right)
$$

Equation (8) can be converted into the following form:

$$
\begin{aligned}
& f(y, \omega)=f^{0}(y, \omega)+\int_{s} S\left(y-y^{\prime}, \omega\right) \mathrm{L}^{1} F\left(\mathrm{y}^{\prime}, \omega\right) \mathrm{d} y^{\prime} \\
& +\rho_{1} \omega^{2} \int_{\mathrm{s}} \mathbf{g}\left(y-y^{\prime}, \omega\right) \mathrm{J} f\left(\mathrm{y}^{\prime}, \omega\right) \mathrm{d} y^{\prime}
\end{aligned}
$$

In which, $\mathrm{S}$ is cylinder cross section, $y=\left(x_{1}, x_{2}\right)$, and

$$
\begin{aligned}
& \mathbf{g}\left(y-y^{\prime}, \omega\right)=\frac{1}{(2 \pi)^{2}} \int_{0}^{\infty} \bar{k} d \bar{k} \quad \bar{k}=\left(k_{1}, k_{2}\right) \\
& \int_{0}^{2 \pi} \mathbf{g}(\bar{k}, \omega) \exp \left(-i \mathrm{~kg}\left(\mathrm{y}-\mathrm{y}^{\prime}\right)\right) d \phi
\end{aligned}
$$

\section{EXPERIMENT RESULT}

While data coding determines the representation of data, modulation determines exactly how tags and readers communicate. RF communications typically consist of a carrier wave modulated to carry data. There are three main classes of digital modulation: Amplitude shift Keying ASK, Frequency shift keying FSK and phase shift keying PSK. Each class has its own power consumption, reliability and bandwidth requirements.

The vast difference in power between tags and readers creates a unique problem for RFID systems. In some differences, the return signal to the reader may be overwhelmed by the outgoing signal, rendering tag responses impossible to detect. To prevent this from occurring, the 
return signal is sometimes modulated onto a different frequency or sub carrier. For example, in ISO 15693 standard for $13.56 \mathrm{MHz}$ RFID, a sub carrier of 13.56 $\mathrm{MHz} / 32=(423.75 \mathrm{KHz})$ is used.

The savant is a middleware system located between a reader and applications in RFID systems. Applications operate on top of, or within the savant operating environment. The savant passes requests from the applications to the reader and receives unique tag identification and possibly other data from sensors, and passes that information to the application. The savant has several fundamental functions integrated into its design some of which are data filtering, aggregation and counting of tag data. These fundamental functions are requiring in order to handle the extremely large quantity of data that RFID systems can generate through the continuous integration of tags.

(1) Object name Service:

The function of the Object Name Service (ONS) in an EPC Network is to identify the location of the server hosting the appropriate information needed by an application. In other words, the ONS acts like a "reverse phone directory" as the ONS uses a number (EPC number) to retrieve the location (of data) from its database. To encourage rapid development of the ONS, the ONS is purely based on existing Internet technology and infrastructure. The first generation of ONS system designs were based upon DNS systems with the first implementations utilizing existing DNS implementations with customized configurations.

(2) EPC Information service:

EPC Information Service (EPCIS) is the gateway between any requester of information and the database. It receives and sends messages in PML with any requester of information, although it's communication with the database can be in any format or standard.

The effect of GSM to RFC is noted on Spectrum analyzer and a Matlab code is developed available at Appendix A. we observed that that the interference of GSM signals to EZF can create a lot of hazard while reading even cause of destroying data elements towards central data base from RFC panel. By only fixing the transmission power to GSM the interference cancellation is possible.

Figure 4 shows the general test configuration. The RFC system consists of a tag, reader and an antenna affixed to RFC reader. The both RFC system and GSM spectra is shown in figure 5 . Figure 6 shows the Restricted power of GSM and RFID at distance d (CL-L(dB)), Pathloss.

\section{DISCUSSION}

From figure we see the first peak of RFC at $868 \mathrm{MHz}$ and second peak is of GSM at $880 \mathrm{MHz}$. When the GSM was not functioning the read range for LTHF RFC is 1.4 $\mathrm{m}$. However, we fixed the distance $\mathrm{D}$ to $1 \mathrm{~m}$, while the power of RFC e.r.p is set to $2 \mathrm{~W}$.

As we know that the coverage level CL of GSM network is $-70 \mathrm{dBm} / 200 \mathrm{KHzf}$ or a big cities and $-80 \mathrm{dBm} / 200$ $\mathrm{KHz}$ for common cities. So we used $-80 \mathrm{dBm} / 200 \mathrm{KHz}$ for our entire simulation of this part. From the equation 4 of model, that shows the relationship between transmission loss and distance from transmitter (d). we can define the transmission loss through equation 4 .

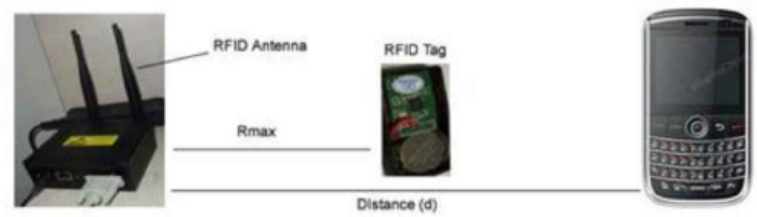

Figure 4. System Compatibility test Environment

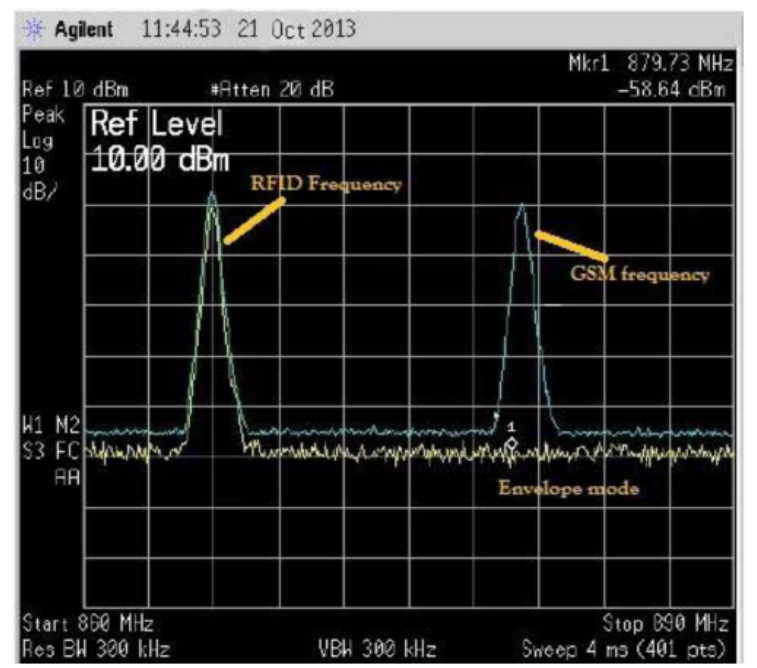

Figure 5. Spectrum of two systems RFID and GSM on different bands

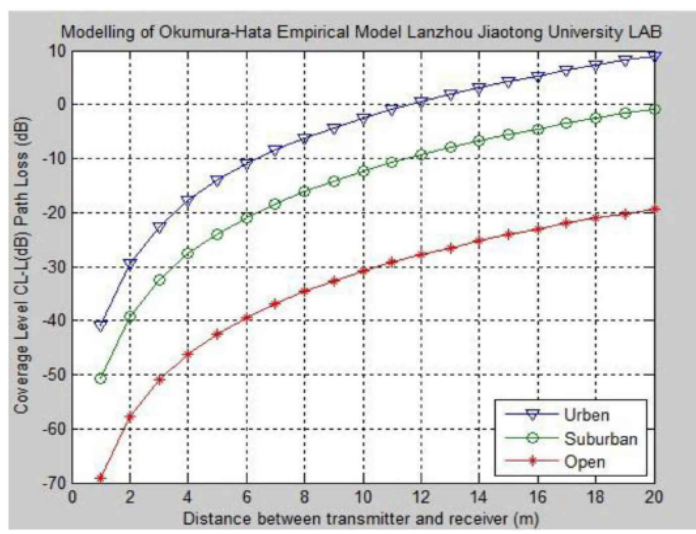

Figure 6. Restricted power of GSM and RFID at distance d (CL$\mathrm{L}(\mathrm{dB}))$, Pathloss.

Figure 6 is a defined relationship between transmission loss and distance $\mathrm{d}$. We see that when distance is $3 \mathrm{~m}$ the RFC power loss is $-34 \mathrm{dBm}$ for big cities and $-47 \mathrm{dBm}$ for common cities as well. So to make sure that RFC equipment can be integrated to GSM equipment, they should be at $-47 \mathrm{~dB}$ restricted power. If the GSM power leaked higher then $-47 \mathrm{dBm}$ the interference between the equipment probably occurs.

In this testing we explain the RFID interference to GSM network. We show that in which conditions GSM and RFID work together, and possible way to eliminate the interference. RFID \& cellular technologies are a promising combination that increases the usability and productivity to provide leading goals. In a simulation part we use Okumura Hata model to see the interference strength at GSM mobile at distance d. According to the given results we should obtained the wasted power on GSM upon RFID 
reader. For instance suppose if there is $3 \mathrm{~m}$ distance of RFID reader and GSM at $880 \mathrm{MHz}$; the restricted power should be $-34 \mathrm{dBm}$ for big cities and $-47 \mathrm{dBm}$ for common cities to avoid interference among two technologies.

Computational testing we check both reader spectrum and fixed spectrum to observe the leaked power behavior quantity. We noticed that if the power is reduced to $47 \mathrm{dBm} / 200 \mathrm{KHz}$; there is no interference among the equipment. So before implanting RFID technology the leaked power should be measured and tested to avoid signal loss. Finally the Okumura Hata model is a significant tool to observe the interference and amount of restricted power.

\section{CONCLUSION}

With increasing interest in deploying RFID system on large scale, problems emerge, and one of them is the reader collision problem. Reader collision problems mainly occur in a dense reader environment, where several readers try to interrogate tags at the same time in same vicinity. Large scale path loss modeling plays a fundamental role in designing both fixed and mobile radio systems. Wireless system is complex systems, so that prediction of the radio coverage is not done in an exact manner. Therefore before setting the system we need to choose a proper model of predicting path loss and interference. These proper methods depend upon the system environment, desire radio coverage and system frequency. The read results can be unsatisfactory with read times and an unacceptable level of misreads. In Europe, the fear of RFID disrupting nonRFID devices operating in the same frequency band as RFID systems has prompted the introduction of the concept of RFID systems. In this paper of predicting path loss and interference analysis of UHF-RFID, we try to eliminate the problems associated of positioning and interference suppression system of library books to RFID systems. Simulation results show that the proposed method can thus improve overall system performance substantially.

\section{REFERENCES}

[1] R. Chedid, F. Mrad, and M. Basma, "Intelligent control of class of wind energy conversion systems," IEEE Trans Energy Convers, pp:1597-1604, 2009.
[2] Y. Errami, M. Ouassaid, and M. Maaroufi, "Modelling and control strategy of PMSG based variable speed wind energy conversion system," In: Proceedings of IEEEICMCS', pp: 1-6, 2011. https://doi.org/10.1109/icmcs.2011.5945736

[3] Y. Wang, et al., "Spectrum Handoff Method Based on Extenics for Cognitive Radio Sensor Networks," International Journal of Online Engineering, vol. 12, no.5, pp. 36-41, May 2016. https://doi.org/10.3991/ijoe.v12i03.5418

[4] R.M. Hilloowala, and A.M. Sharaf, "A rule-based fuzzy logic controller for a PWM inverter in a standalone wind energy conversion scheme," IEEE Trans Ind Appl, pp:57-65, 2006.

[5] K. Johnson, et al., "Methods for increasing region 2 power capture on a variable speed wind turbine," J Solar Energy Eng, pp:10921100, 2004.. https://doi.org/10.1115/1.1792653

[6] K.E. Johnson, L.Y. Pao, M.J. Balas, et al., "Control of variablespeed wind turbines: Standard and adaptive techniques for maximizing energy capture," IEEE Control Syst Mag, pp:70-81, 2006.

[7] S. Müller, M. Deicke, and R.W. De Doncker, "Doubly fed induction generator system for wind turbines," IEEE Ind Appl Mag, pp:26-33, 2002. https://doi.org/10.1109/2943.999610

[8] Q. Wang, amd L.C. Chang, "An intelligent maximum power extraction algorithm for inverter based variable speed wind turbine systems". IEEE Trans Power Electron, pp:1242-1249, 2012.

[9] Z. Chen, J.M. Guerrero, and F. Blaabjerg, "A review of the state of the art of power electronics for wind turbines," IEEE Trans Power Electron, pp:1859-1875, 2009. https://doi.org/10.1109/ TPEL.2009.2017082

[10] H. Jing, "Node deployment algorithm based on perception model of wireless sensor network," International Journal of Automation Technology, vol.9, no.3, pp. 210-215, April 2015. https://doi.org/10.20965/ijat.2015.p0210

[11] H. Jing,"Routing optimization algorithm based on nodes density and energy consumption of wireless sensor network," Journal of Computational Information Systems, vol. 11, no.14, pp. 50475054, July 2015.

\section{AUTHORS}

Zhang Xiao-guang, Zhao Yi-jie, and Zhao Wei are with Hebei North University, Hebei, China (e-mail: 404542905@qq.com).

The paper is supported by 2013 Hebei North University surfaces university research project. Submitted 09 September 2016. Published as resubmitted by the authors 23 October 2016 . 